\title{
A narrative review of mechanisms of ferroptosis in cancer: new challenges and opportunities
}

\author{
Mingyan Jiang ${ }^{1,2}$, Ruolan $\mathrm{Hu}^{1,2}$, Ruixin Yu${ }^{1,2}$, Yiwei Tang ${ }^{1,2}$, Jinrong $\mathrm{Li}^{1,2}$ \\ ${ }^{1}$ Department of Pediatrics, West China Second University Hospital, Sichuan University, Chengdu, China; ${ }^{2}$ Key Laboratory of Birth Defects and \\ Related Diseases of Women and Children (Sichuan University), Ministry of Education, Chengdu, China \\ Contributions: (I) Conception and design: M Jiang, J Li; (II) Administrative support: J Li; (III) Provision of study materials or patients: M Jiang, R Hu, \\ R Yu, Y Tang; (IV) Collection and assembly of data: M Jiang, J Li; (V) Data analysis and interpretation: M Jiang, J Li; (VI) Manuscript writing: All \\ authors; (VII) Final approval of manuscript: All authors. \\ Correspondence to: Jinrong Li. Department of Pediatrics, West China Second University Hospital, Sichuan University, Chengdu, China. \\ Email: ljinrong224@yeah.net.
}

Objective: This article reviews the specific mechanism of ferroptosis in cancer and introduces in detail the opportunities and challenges of ferroptosis-based cancer therapy, aiming to provide a new research direction for tumor therapy.

Background: Ferroptosis is a newly discovered programmed non-apoptotic form of cell death. Involving changes in metabolic processes and the accumulation of peroxidation caused by factors such as drugs or genes which destruct the cell membrane structure, this kind of cell death has been linked with the pathological process of diseases such as tumors, neurological diseases, ischemia-reperfusion injury, kidney injury, and hemopathy. This kind of cell death can play a vital role in inhibiting tumorigenesis by eliminating the adaptive characteristics of malignant cells and removing cells that are unable to obtain key nutritional factors or are infected and damaged by environmental changes. The present focus of research on the regulation of ferroptosis-related diseases involves the use of small molecule compounds.

Methods: We described the mechanism of ferroptosis and its related small molecules compounds, which involved in the regulatory mechanism, and analyzed the role and regulatory mechanism of ferroptosis in different tumors.

Conclusions: This article reviewed the mechanism of ferroptosis and its role and mechanism in different tumors, and showed it can inhibit the occurrence and development of different tumors and may reduce the adverse effects of current treatment methods.

Keywords: Ferroptosis; cancer; mechanism of action

Submitted Aug 30, 2021. Accepted for publication Sep 28, 2021.

doi: $10.21037 /$ atm-21-4863

View this article at: https://dx.doi.org/10.21037/atm-21-4863

\section{Introduction}

Cell death is an irreversible process closely related to the growth, development, aging, and disease of organisms, and research has shown a variety of programmed cell death (PCD) forms such as apoptosis, necrosis, pyroptosis, and oxeiptosis. Ferroptosis was discovered in 2012 (1), and unlike other PCD, is characterized by the absence of apoptosis and dependence on iron, which can disrupt the intracellular redox balance (2). Cells undergoing ferroptosis show unique morphological characteristics, such as mitochondrial atrophy and an increased density of the mitochondrial membrane (3). Ferroptosis is accompanied by a disturbance of intracellular iron ion current and a significant increase of reactive oxygen species (ROS) and lipid peroxidation, the latter of which in the inner cell 
membrane causes membrane damage, and ultimately prompts cell disintegration and death. Ferroptosis has been confirmed to be closely related to various diseases such as neurodegenerative diseases, tumors, cardiovascular and cerebrovascular diseases, and acute kidney injury (4), and can be caused by different physiological and pathological stress conditions in both humans and animals (3). This kind of cell death can play a vital role in inhibiting tumorigenesis by eliminating the adaptive characteristics of malignant cells and removing cells that are unable to obtain key nutritional factors or are infected and damaged by environmental changes (5). This article reviews the specific mechanism of ferroptosis in cancer and introduces in detail the opportunities and challenges of ferroptosis-based cancer therapy, aiming to provide a new research direction for tumor therapy. We present the following article in accordance with the Narrative Review reporting checklist (available at https://dx.doi.org/10.21037/atm-21-4863).

\section{Mechanism of ferroptosis}

Ferroptosis is characterized by the intracellular irondependent accumulation of lipid peroxides (6), which is known to be influenced by iron ions, lipid reactive oxygen species (L-ROS), and glutathione peroxidase 4 (GPX4). It has been widely shown that a crucial cause of ferroptosis is the excessive accumulation of L-ROS, which depends on the catalysis of intracellular iron ions. This results in a break in the balance of the production and clearance of ROS, which ultimately leads to ferroptosis $(3,7,8)$. Intracellular ROS are mainly produced by the mitochondrial respiratory chain, and can also be produced by a series of chemical reactions in endoplasmic reticulum, reduced nicotinamide adenine dinucleotide phosphate (NADPH) and NADPH oxidase (NOX). Normally, the oxidation-antioxidant system maintains ROS in a certain range, with beyond the normal range considered as promoting tumor development and progression. GPX4, heat shock factor-binding protein $\beta 1$ (HSBP1), and nuclear factor-factor 2 (Nrf2) negatively regulate ferroptosis by limiting ROS production and reducing cellular uptake of iron. By contrast, NADPH oxidase and P53 exert positive regulation by promoting ROS production (9) (Figure 1).

\section{Lipid peroxidation}

The main characteristic of ferroptosis is the iron-dependent accumulation of lipid peroxides accompanied by a deficiency of oxidoreductase. Lipids maintain the integrity of cell membranes, and destruction of the redox balance in biological systems contributes to extensive lipid peroxidation which alters the assembly, composition, structure, and dynamics of lipid membranes. Lipid peroxides can also further generate ROS or degrade into active compounds responsible for DNA-protein cross-linking, causing damage to skin, nerves, tissues, and organs (10). Lipid peroxidation and cellular antioxidant capacity are also critical regulatory targets for the cystine/glutamate antiporter system Xc- and GPX4 (9). System Xc- is an upstream node of the GPX4GSH-cysteine axis and a key factor in ferroptosis (11), while erastin promotes ferroptosis by directly inhibiting the activity of the cystine/glutamate antiporter system Xc$(12,13)$. On the one hand, the inhibition of system Xcinhibits the uptake of cysteine and reduces the synthesis of glutathione (GSH), leading to GSH depletion in cancer cells. GSH is a reducing co-substrate of GPX4 and an essential antioxidant in cells which it protects from oxidative damage caused by highly toxic ROS. However, depletion of GSH results in the inactivation of GPX4 and promotes the accumulation of ROS in cancer cells causing their death $(8,9,14)$. On the other hand, inhibition of system Xc- also inactivates the cystine-cysteine redox cycle, restricting the supply of intracellular cysteine and further inhibiting the synthesis of GSH, which ultimately leads to ferroptosis $(15,16)$.

\section{Iron metabolism}

Genes related to iron metabolism are confirmed to be key mediators of ferroptosis (Figure 1). After binding to transferrin receptor 1 (TFR1), $\mathrm{Fe}^{3+}$ ions enter the endosome through the cell membrane and localize in the endoderm, where they are reduced to divalent ions by ferric reductase [six-transmembrane epithelial antigen of the prostate (STEAP)] (17). $\mathrm{Fe}^{2+}$ is then promoted to release into the labile iron pool in the cytoplasm, and excess iron is stored in ferritin. Ferroportin is an iron export protein also known as SLC11A3, which oxidizes $\mathrm{Fe}^{2+}$ to $\mathrm{Fe}^{3+}$ for export. Divalent metal transporter 1 (DMT1 or SLC11A2) releases $\mathrm{Fe}^{2+}$ from the nucleus into a cytoplasmic labile iron pool, where intracellular overloaded $\mathrm{Fe}^{2+}$ catalyzes the production of excessive ROS through the Fenton reaction (14), affecting the state of the cell. Excess iron is stored intracellularly as ferritin light chain (FTL) and ferritin heavy chain 1 (FTH1) (18). While studies have shown that increasing exogenous iron ions can accelerate erastin-induced ferroptosis, 


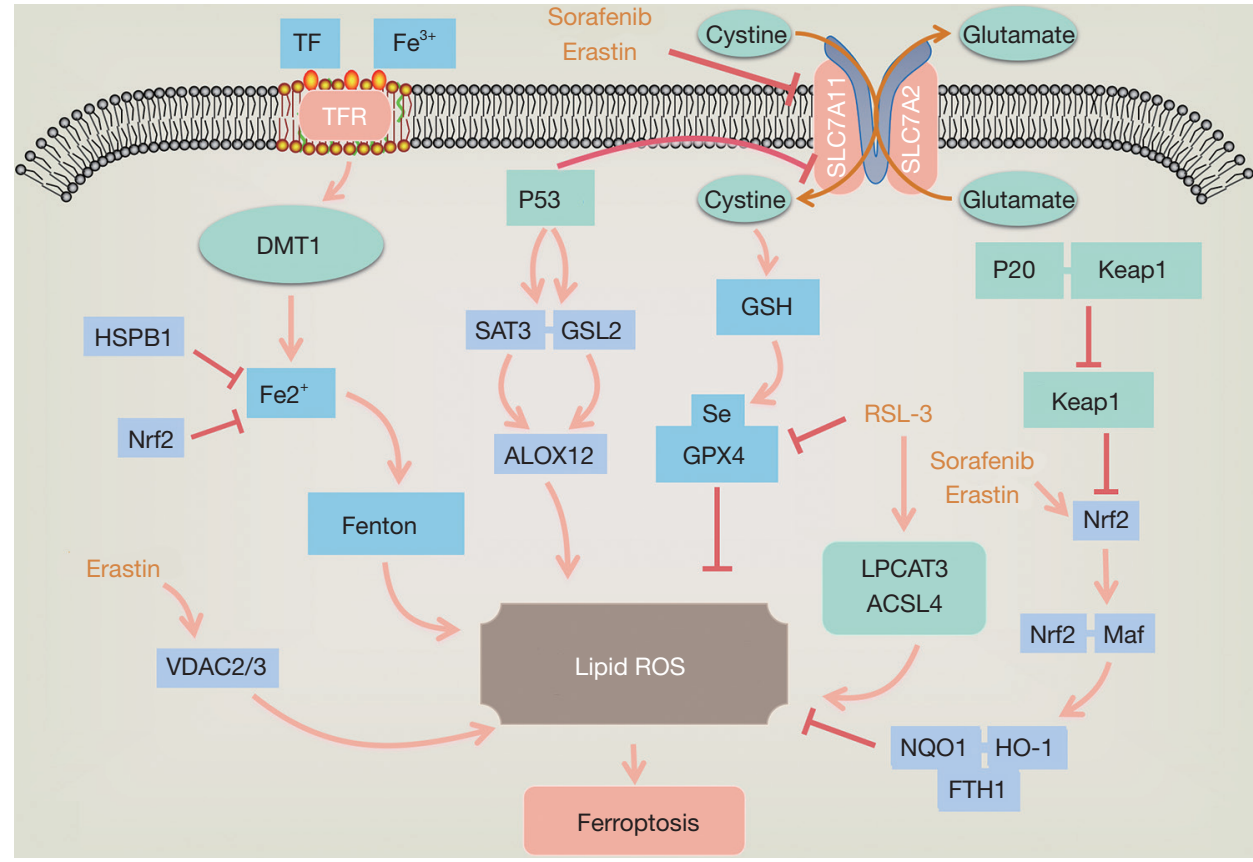

Figure 1 Mechanism of ferroptosis.

the addition of other divalent metal ions has no such effect (3). In another study on the high-throughput screening of small molecules in 2008, RSL3 was found to directly inhibit the activity of GPX4 and selectively kill BJeLR cells in the form of ferroptosis. However, antioxidants and iron chelators could block RSL3-induced ferroptosis, further suggesting that ferroptosis is an iron-dependent cell death (19) (Figure 1). Ferroptosis in RAS-mutant cancer cells with mutant $R A S$ also showed significantly increased cellular iron intake but reduced storage capacity (20). The above findings prove that the regulation of iron metabolism pathways can lead to increased iron content in tumor cells, making cancer tissues more sensitive to ferroptosis, which indicates iron is essential for ferroptosis.

\section{P53 gene}

The P53 gene is a tumor suppressor gene which mediates cell cycle arrest, senescence, and apoptosis which affect tumor development, and some studies have indicated that it is involved in ferroptosis (Figure 1). By directly inhibiting the transcription of SLC7A11, a key component of system $\mathrm{Xc-}$, P 53 can inhibit the cellular uptake of cystine, and can also participate in ferroptosis in colon cancer cells by regulating Yes-associated protein 1 (YAP1), contributing to the inhibition of colon cancer and its treatment (21). During ferroptosis, P53 achieves its effects on gene metabolism, post-translational regulation, and the $\mathrm{P} 53-\mathrm{p} 21$ axis through various mechanisms (22), and under specific conditions, can promote, limit, or delay ferroptosis. However, the bidirectional regulation of ferroptosis by P53 in a cellspecific or context-dependent manner requires further study.

\section{MicroRNAs (miRNAs)}

miRNAs are small, single-stranded, non-coding RNAs that can bind to mRNAs to negatively regulate target gene expression. SLC1A5-mediated glutamine transport plays a key role in tumor cell metabolism, proliferation, and ferroptosis, and its inhibition can be used as a potential approach for solid tumor therapy. Specifically, miR-137 can negatively regulate ferroptosis by directly targeting SLC1A5 (23). MiR-13 overexpression inhibits the production of SLC1A5, resulting in a reduction in cellular glutamine uptake and accumulation of malondialdehyde (MDA). This causes endogenous miR-137 inactivation using related antagonists, and tumor cells show up-regulated sensitivity to erastin-induced ferroptosis. Sangokoya et al. (24) found that miR-485-3P and miR-194 target 
and regulate the expression of ferroportin by adding exogenous iron and defetoxamine (DFO). Cancer cells can achieve the accumulation of iron content by largely inducing miR-485-3P and reducing intracellular ferroportin. Additionally, miRNA-7 and miRNA200a can activate the Nrf2 pathway by inhibiting the expression of Keap1 (25), while miRNA-28 inhibits Nrf2 in a Keap1-independent manner to affect cellular ferroptosis (26). Both miRNA-101 and miRNA-455 can promote Nrf2 expression by targeting Cullin-3 (27), while miRNA-153, miRNA-142-5p, miRNA-27a, miRNA-144, miRNA-93, miRNA-34a, miRNA-365-1, miRNA-193b, and miRNA-29-b1 (27) can further affect ferroptosis by reducing Nrf2 through different mechanisms.

\section{Other mechanisms}

Studies have reported that the voltage-dependent anion channel (VDAC) 2 (VDAC2)/VDAC 3 pathway controls the transmembrane flow of adenosine diphosphate (ADP), phosphatidylinositol (PI), and adenosine triphosphate (ATP) on the mitochondrial outer membrane, and that tubulin can inhibit mitochondrial metabolism by blocking the VDAC2/ VDAC3 pathway, promoting aerobic glycolysis which is the main energy source for tumor cells. Erastin, on the other hand, can induce ferroptosis by inhibiting cystine/ glutamate antiporters by binding to VDAC2, VDAC3, and SLC7A5 (28). Additionally, in a U.S study it was found that the long-chain fatty acyl-CoA synthetase family member 4 (ACSL4), which is related to lipid metabolism, is one of the key proteins in ferroptosis (29). Arachidonic acid and adrenal acid are polyunsaturated fatty acid substrates. They can be acylated by ACSL4 and become part of membrane phospholipids, which can be easily oxidized to L-ROS and reduced by GSH and GPX4. RSL3 can directly inhibit GPX4, leading to the accumulation and L-ROS and lipid peroxidation, consequently triggering ferroptosis (29) (Figure 1). In addition, Cysteinyl-tRNA synthetase can deprive cystine, reduce the synthesis of GSH, and inactivate GPX4, producing ROS to induce ferroptosis (30).

\section{Regulatory mechanism of ferroptosis in different tumors}

Cancer is ranked by the World Health Organization as the second leading cause of death and can occur in almost any organ or tissue (31). As a novel mode of cell death discovered in recent years, ferroptosis has been reported in colorectal cancer (32), gastric cancer (33), pancreatic cancer (34), lung cancer (35), prostate cancer, and glioma (4). Researchers have shown that inducing ferroptosis or using ferroptosis activators in combination with anti-cancer drugs is conducive to the intervention, inhibition, and clearance of cancer. Changes in iron metabolism mechanisms, especially in iron intake and export, contribute to the occurrence and metastasis of cancer (36). An increase in the number of TFR1, decrease in ferroportin levels, and high levels of hepcidin have been seen on the surface of various tumor cells including liver, breast, and prostate cancer, which reduce the efflux of intracellular iron while promoting extracellular iron intake (37-39). At the same time, tumor tissues with intact basement membrane are found in an ischemia-hypoxic state during proliferation, and a longterm hypoxic state can induce tumor cells to express a series of hypoxia inducible factors (HIF) (40). The activation of HIF can activate the transcription of multiple genes related to tumorigenesis such as vascular endothelial growth factor A and zinc-finger proteins, and can also promote iron intake, affecting ferroptosis (41). Therefore, tumor cells can achieve increased intracellular iron content by regulating iron metabolism pathways, which makes cancer tissues more sensitive to ferroptosis.

\section{Hepatocellular carcinoma (HCC)}

The results of traditional treatment for HCC, including surgery and chemotherapy, are unsatisfactory, especially for advanced HCC. Ferroptosis is a newly discovered mechanism of cell death and a potential biomarker for HCC treatment (42), and according to relevant studies, systemic and intracellular iron homeostasis are altered in HCC and many cancer subtypes (36). Abnormal iron absorption (43) and a disturbance of lipid metabolism are observed in HCC (44). CircRNA0097009 affects HCC by sponging miR-1261 and acting as a competitive endogenous RNA to regulate SLC7A11 expression and to induce ferroptosis (44). GSTZ1 also makes HCC cells sensitive to sorafenib-induced ferroptosis by inhibiting the NRF2/GPX4 axis (45). In addition, the p62-Keap1NRF2 pathway plays a role in ferroptosis of HCC cells, and inhibition of NRF2 expression can up-regulate iron and ROS levels enhancing the antitumor effect of erastin (46). Iron and L-ROS, by definition and process of ferroptosis, are vital factors mediating it, as is GPX4. Researchers have elaborated the relationship of ferroptosis with the three key factor through different perspectives (such as tumor 
suppressor genes, non-coding genes, regulatory proteins, signaling pathways, and small molecule compounds) (43). Thus, the proposal of ferroptosis provides new ideas for the treatment of HCC.

\section{Gastric cancer}

Gastric cancer is currently the fifth most common and third most deadly malignancy in the world (47). While traditional radical surgery and palliative chemotherapy remain the most common treatment, and cisplatin-based chemotherapy is the most used first-line treatment, patients with advanced gastric cancer usually develop drug resistance, resulting in a poor prognosis (48). There is currently evidence that ferroptosis is associated with chemoresistance in gastric cancer (49). Elevated ATF3 has been shown to induce ferroptosis in gastric cancer cells by inhibiting the Nrf2/ Keap1/xCT signaling pathway, consequently alleviating cisplatin resistance (50). Erastin has also been reported to induce ferroptosis in gastric cancer cells, while ferroptosis inhibitors (F-1 and L-1) can inhibit cysteine dioxygenase I (CDO1), restoring GPX4 expression and GSH levels and ultimately reducing ROS generation to reverse erastin induced ferroptosis (51). CD44 is an adhesion molecule expressed in cancer stem cells, and its mutant (CD44v) can interact with system Xc- to regulate intracellular GSH. A high CD44 expression in human gastrointestinal tumor cells then indicates high GSH synthesis capacity, which in turn can block ROS-induced stress signal transduction and make gastric cancer cells resistant to ferroptosis (52).

\section{Ovarian cancer}

Ovarian cancer is the deadliest malignancy in females, killing about 150,000 women each year (53). As this cancer has no obvious and typical clinical symptoms in its early stages, about $75 \%$ patients are diagnosed at an advanced stage, and between $70-80 \%$ will relapse after treatment (54), with a 5 -year survival rate of approximately $25-35 \%$ (55). As advanced patients will develop drug resistance (56), there is an urgent need for new treatments. Studies have shown that continuous iron stimulation is a high-risk factor for ovarian cancer (57). In a study by Yamada et al. (58), following treatment of the normal ovarian cell line OSE/E7/human telomerase reverse transcriptase (hTERT) genome with iron ions, the genome showed the characteristics of ovarian cancer, and normal ovarian cells gradually transformed into cancer cells. Lidocaine can also promote ferroptosis in ovarian cancer by targeting the miR-382-5p/SLC7A11 axis (59), as does superparamagnetic iron oxide cultured in human serum by regulating p53 (60). Additionally, as being a well-tolerated anti-malarial drug, artesunate can also promote ROS and iron-dependent cell death in ovarian cancer cells, but this process can be prevented by the ferroptosis inhibitor F-1 (61). Thus, ferroptosis may be crucial in the treatment of ovarian cancer.

\section{Breast cancer}

Breast cancer is the leading cause of death in women worldwide and has a poor prognosis, especially triplenegative breast cancer (TNBC). TNBC is an aggressive breast cancer with a higher recurrence mortality rate than other types (62). While L-cysteine deficiency in TNBC is associated with high lethality, the ferroptosis inhibitors DFO and F-1 can hinder cell death caused by L-cysteine deficiency, suggesting it can induce ferroptosis in TNBC cells (63). Ferroptosis can be induced by increasing irondependent ROS (64), and CDO1 overexpression decreases GSH levels in breast cancer cells, leading to further accumulation of ROS and accelerating the occurrence of ferroptosis (65). SAS triggers ferroptosis in breast cancer ZR-75-1 cells by inhibiting the expression of $\mathrm{xCT}$ protein and GPX4 protein, which are functional subunits of system Xc-, and by promoting DMT1 expression (44). Additionally, metformin induces ferroptosis in breast cancer by targeting the miR-324-3p/GPX4 axis (66) and by inhibiting UFMylation of SLC7A11 (67).

\section{Lung cancer}

Lung cancer is the malignant tumor with the highest global mortality rate, accounting for approximately $25 \%$ of cancer deaths. Since lung cancer is a highly heterogeneous tumor with complex molecular mechanisms (68), many molecularly targeted drugs are ineffective for some patients, posing a great challenge for its treatment. According to a recent clinical study, serum iron, ferritin, and total iron binding capacity are significantly higher in lung cancer patients than in healthy controls, and the higher the serum iron concentration, the higher the risk of lung cancer (69). Consistent with these results, data from a Taiwanese study also showed that high serum iron $(>120 \mu \mathrm{g} / \mathrm{dL})$ increased the risk of incidence and mortality from malignant tumors, presenting a positively correlation (70). In addition, cisplatin combined with erastin has a significant synergistic effect 
on anti-tumor activity in the treatment of lung cancer (71). Cysteine desulfurase (NFS1) is responsible for the biosynthesis of iron-sulfur clusters and can protect cells from ferroptosis under high oxygen tension by maintaining iron-sulfur cofactors, while the inhibition of GPX4 or mTOR can overcome resistance to lapatinib by promoting ferroptosis in lung cancer cells (72). YTHDC2 can act as an endogenous ferroptosis inducer in lung cancer, and SLC7A11, the main functional subunit of system XC-, is essential in this process (73). Collectively, these results show ferroptosis plays a critical role in lung cancer.

\section{Conclusions and prospects}

As a newly discovered non-PCD, ferroptosis is closely related to a variety of physiopathological processes, and currently holds an essential place in research on new therapeutic strategies for cancer. This article reviewed the mechanism of ferroptosis and its role and mechanism in different tumors, and showed it can inhibit the occurrence and development of different tumors and may reduce the adverse effects of current treatment methods. It has been reported that the acquisition of mesenchymal states, such as epithelial-mesenchymal transformation or cancer stem cells, is closely related to tumor cell metastasis and spread, and to drug resistance and chemotherapy resistance (74). A highmesenchymal state is an important mechanism in acquired and non-acquired anti-targeted therapy. The survival of tumor cells with a high-mesenchymal state depends on GPX4, which is closely related to ferroptosis and indicates its critical involvement in tumors. In addition, some studies have found that ferroptosis is more immunogenic than apoptosis, and cells undergoing ferroptosis release inflammatory-related injury molecules [such as high mobility group protein 1 (HMGB1)], promoting inflammation through danger-associated molecular patterns (DAMPs) (75). Ferroptosis can also recruit and activate immune cells at the tumor site by transmitting chemotactic signals, indicating that ferroptosis inducers are likely to promote anti-tumor immunotherapy.

Although ferroptosis is effective in cancer treatment, there remain many questions, which require further investigation. These include, how to explain the relationship between ferroptosis and other cell death processes at the molecular level; whether ferroptosis can enhance the immunogenicity of cells to the host, thereby causing adaptive immune responses; and what specific types of oxidized lipids are involved in ferroptosis. In addition, in depth studies on the mechanism of ferroptosis and its feasibility in the clinical treatment of tumors are required.

\section{Acknowledgments}

Funding: This study was supported by the Application Foundation Program of Science and Technology Department of Sichuan Province (2021YJ0453), the Youth Innovation Project of Medical Research in Sichuan Province (Grant No. Q18015), and the Health and Family Planning Commission of Sichuan Province (Grant No. 150106).

\section{Footnote}

Reporting Checklist: The authors have completed the Narrative Review reporting checklist. Available at https:// dx.doi.org/10.21037/atm-21-4863

Conflicts of Interest: All authors have completed the ICMJE uniform disclosure form (available at https://dx.doi. org/10.21037/atm-21-4863). The authors have no conflicts of interest to declare.

Ethical Statement: The authors are accountable for all aspects of the work in ensuring that questions related to the accuracy or integrity of any part of the work are appropriately investigated and resolved.

Open Access Statement: This is an Open Access article distributed in accordance with the Creative Commons Attribution-NonCommercial-NoDerivs 4.0 International License (CC BY-NC-ND 4.0), which permits the noncommercial replication and distribution of the article with the strict proviso that no changes or edits are made and the original work is properly cited (including links to both the formal publication through the relevant DOI and the license). See: https://creativecommons.org/licenses/by-nc-nd/4.0/.

\section{References}

1. Dolma S, Lessnick SL, Hahn WC, et al. Identification of genotype-selective antitumor agents using synthetic lethal chemical screening in engineered human tumor cells. Cancer Cell 2003;3:285-96.

2. Zuo S, Yu J, Pan H, et al. Novel insights on targeting ferroptosis in cancer therapy. Biomark Res 2020;8:50.

3. Dixon SJ, Lemberg KM, Lamprecht MR, et al. Ferroptosis: an iron-dependent form of nonapoptotic cell 
death. Cell 2012;149:1060-72.

4. Fonseca-Nunes A, Jakszyn P, Agudo A. Iron and cancer risk--a systematic review and meta-analysis of the epidemiological evidence. Cancer Epidemiol Biomarkers Prev 2014;23:12-31.

5. Rhodes SL, Buchanan DD, Ahmed I, et al. Pooled analysis of iron-related genes in Parkinson's disease: association with transferrin. Neurobiol Dis 2014;62:172-8.

6. Sha W, Hu F, Xi Y, et al. Mechanism of Ferroptosis and Its Role in Type 2 Diabetes Mellitus. J Diabetes Res 2021;2021:9999612.

7. Guiney SJ, Adlard PA, Bush AI, et al. Ferroptosis and cell death mechanisms in Parkinson's disease. Neurochem Int 2017;104:34-48.

8. Yang WS, SriRamaratnam R, Welsch ME, et al. Regulation of ferroptotic cancer cell death by GPX4. Cell 2014;156:317-31

9. Xie Y, Hou W, Song X, et al. Ferroptosis: process and function. Cell Death Differ 2016;23:369-79.

10. Gaschler MM, Stockwell BR. Lipid peroxidation in cell death. Biochem Biophys Res Commun 2017;482:419-25.

11. Chen L, Qiao L, Bian Y, et al. GDF15 knockdown promotes erastin-induced ferroptosis by decreasing SLC7A11 expression. Biochem Biophys Res Commun 2020;526:293-9.

12. Huo H, Zhou Z, Qin J, et al. Erastin Disrupts Mitochondrial Permeability Transition Pore (mPTP) and Induces Apoptotic Death of Colorectal Cancer Cells. PLoS One 2016;11:e0154605.

13. Dächert J, Schoeneberger H, Rohde K, et al. RSL3 and Erastin differentially regulate redox signaling to promote Smac mimetic-induced cell death. Oncotarget 2016;7:63779-92.

14. Angeli JPF, Shah R, Pratt DA, et al. Ferroptosis Inhibition: Mechanisms and Opportunities. Trends Pharmacol Sci 2017;38:489-98.

15. Banjac A, Perisic T, Sato H, et al. The cystine/cysteine cycle: a redox cycle regulating susceptibility versus resistance to cell death. Oncogene 2008;27:1618-28.

16. Dixon SJ, Patel DN, Welsch M, et al. Pharmacological inhibition of cystine-glutamate exchange induces endoplasmic reticulum stress and ferroptosis. Elife 2014;3:e02523.

17. Ding H, Chen S, Pan X, et al. Transferrin receptor 1 ablation in satellite cells impedes skeletal muscle regeneration through activation of ferroptosis. J Cachexia Sarcopenia Muscle 2021;12:746-68.

18. Chen P, Li FM, Zhou YF, et al. Effects of alpha-lipoic acid on expression of iron transport and storage proteins in BV-2 microglia cells. Pharmacol Rep 2017;69:1-5.

19. Yang WS, Stockwell BR. Synthetic lethal screening identifies compounds activating iron-dependent, nonapoptotic cell death in oncogenic-RAS-harboring cancer cells. Chem Biol 2008;15:234-45.

20. Manz DH, Blanchette NL, Paul BT, et al. Iron and cancer: recent insights. Ann N Y Acad Sci 2016;1368:149-61.

21. Ye S, Xu M, Zhu T, et al. Cytoglobin promotes sensitivity to ferroptosis by regulating p53-YAP1 axis in colon cancer cells. J Cell Mol Med 2021;25:3300-11.

22. Li T, Kon N, Jiang L, et al. Tumor suppression in the absence of p53-mediated cell-cycle arrest, apoptosis, and senescence. Cell 2012;149:1269-83.

23. Luo $\mathrm{M}, \mathrm{Wu} \mathrm{L}$, Zhang $\mathrm{K}$, et al. miR-137 regulates ferroptosis by targeting glutamine transporter SLC1A5 in melanoma. Cell Death Differ 2018;25:1457-72.

24. Sangokoya C, Doss JF, Chi JT. Iron-responsive miR485-3p regulates cellular iron homeostasis by targeting ferroportin. PLoS Genet 2013;9:e1003408.

25. Kabaria S, Choi DC, Chaudhuri AD, et al. MicroRNA-7 activates Nrf2 pathway by targeting Keap1 expression. Free Radic Biol Med 2015;89:548-56.

26. Yang M, Yao Y, Eades G, et al. MiR-28 regulates Nrf2 expression through a Keap1-independent mechanism. Breast Cancer Res Treat 2011;129:983-91.

27. Xu D, Zhu H, Wang C, et al. microRNA-455 targets cullin 3 to activate Nrf2 signaling and protect human osteoblasts from hydrogen peroxide. Oncotarget 2017;8:59225-34.

28. Li M, Wang X, Lu S, et al. Erastin triggers autophagic death of breast cancer cells by increasing intracellular iron levels. Oncol Lett 2020;20:57.

29. Skouta R, Dixon SJ, Wang J, et al. Ferrostatins inhibit oxidative lipid damage and cell death in diverse disease models. J Am Chem Soc 2014;136:4551-6.

30. Hayano M, Yang WS, Corn CK, et al. Loss of cysteinyltRNA synthetase (CARS) induces the transsulfuration pathway and inhibits ferroptosis induced by cystine deprivation. Cell Death Differ 2016;23:270-8.

31. World Health Organizatio. World Health Organization/ international Collaborative Study of Medical Care Utilization. 1970.

32. Yu H, Guo P, Xie X, et al. Ferroptosis, a new form of cell death, and its relationships with tumourous diseases. J Cell Mol Med 2017;21:648-57.

33. Sharma AK, Bhattacharya S, Khan SA, et al. Dynamic alteration in $\mathrm{H} 3$ serine 10 phosphorylation is G1-phase specific during ionization radiation induced DNA damage 
response in human cells. Mutat Res 2015;773:83-91.

34. Yamaguchi Y, Kasukabe T, Kumakura S. Piperlongumine rapidly induces the death of human pancreatic cancer cells mainly through the induction of ferroptosis. Int J Oncol 2018;52:1011-22.

35. Alvarez SW, Sviderskiy VO, Terzi EM, et al. NFS1 undergoes positive selection in lung tumours and protects cells from ferroptosis. Nature 2017;551:639-43.

36. Torti SV, Torti FM. Iron and cancer: more ore to be mined. Nat Rev Cancer 2013;13:342-55.

37. Jeong SM, Lee J, Finley LW, et al. SIRT3 regulates cellular iron metabolism and cancer growth by repressing iron regulatory protein 1 . Oncogene 2015;34:2115-24.

38. Daniels TR, Bernabeu E, Rodríguez JA, et al. The transferrin receptor and the targeted delivery of therapeutic agents against cancer. Biochim Biophys Acta 2012;1820:291-317.

39. Jeong SM, Hwang S, Seong RH. Transferrin receptor regulates pancreatic cancer growth by modulating mitochondrial respiration and ROS generation. Biochem Biophys Res Commun 2016;471:373-9.

40. Nozawa-Suzuki N, Nagasawa H, Ohnishi K, et al. The inhibitory effect of hypoxic cytotoxin on the expansion of cancer stem cells in ovarian cancer. Biochem Biophys Res Commun 2015;457:706-11.

41. Sass G, Barikbin R, Tiegs G. The multiple functions of heme oxygenase-1 in the liver. Z Gastroenterol 2012;50:34-40.

42. Deng T, Hu B, Jin C, et al. A novel ferroptosis phenotyperelated clinical-molecular prognostic signature for hepatocellular carcinoma. J Cell Mol Med 2021;25:6618-33.

43. Chen J, Chloupková M. Abnormal iron uptake and liver cancer. Cancer Biol Ther 2009;8:1699-708.

44. Lyu N, Zeng Y, Kong Y, et al. Ferroptosis is involved in the progression of hepatocellular carcinoma through the circ0097009/miR-1261/SLC7A11 axis. Ann Transl Med 2021;9:675.

45. Wang Q, Bin C, Xue Q, et al. GSTZ1 sensitizes hepatocellular carcinoma cells to sorafenib-induced ferroptosis via inhibition of NRF2/GPX4 axis. Cell Death Dis 2021;12:426.

46. Sun X, Ou Z, Chen R, et al. Activation of the p62Keap1-NRF2 pathway protects against ferroptosis in hepatocellular carcinoma cells. Hepatology 2016;63:173-84.

47. Venerito M, Vasapolli R, Rokkas T, et al. Gastric cancer: epidemiology, prevention, and therapy. Helicobacter 2018;23 Suppl 1:e12518.

48. Zhao TT, Xu H, Xu HM, et al. The efficacy and safety of targeted therapy with or without chemotherapy in advanced gastric cancer treatment: a network meta-analysis of well-designed randomized controlled trials. Gastric Cancer 2018;21:361-71.

49. Guo J, Xu B, Han Q, et al. Ferroptosis: A Novel Anti-tumor Action for Cisplatin. Cancer Res Treat 2018;50:445-60.

50. Fu D, Wang C, Yu L, et al. Induction of ferroptosis by ATF3 elevation alleviates cisplatin resistance in gastric cancer by restraining Nrf2/Keap1/xCT signaling. Cell Mol Biol Lett 2021;26:26.

51. Hao S, Yu J, He W, et al. Cysteine Dioxygenase 1 Mediates Erastin-Induced Ferroptosis in Human Gastric Cancer Cells. Neoplasia 2017;19:1022-32.

52. Ishimoto T, Nagano O, Yae T, et al. CD44 variant regulates redox status in cancer cells by stabilizing the $\mathrm{xCT}$ subunit of system xc(-) and thereby promotes tumor growth. Cancer Cell 2011;19:387-400.

53. Lheureux S, Gourley C, Vergote I, et al. Epithelial ovarian cancer. Lancet 2019;393:1240-53.

54. Matulonis UA, Sood AK, Fallowfield L, et al. Ovarian cancer. Nat Rev Dis Primers 2016;2:16061.

55. Lee JY, Kim S, Kim YT, et al. Changes in ovarian cancer survival during the 20 years before the era of targeted therapy. BMC Cancer 2018;18:601.

56. Salani R, Khanna N, Frimer M, et al. An update on post-treatment surveillance and diagnosis of recurrence in women with gynecologic malignancies: Society of Gynecologic Oncology (SGO) recommendations. Gynecol Oncol 2017;146:3-10.

57. Lattuada D, Uberti F, Colciaghi B, et al. Fimbrial cells exposure to catalytic iron mimics carcinogenic changes. Int J Gynecol Cancer 2015;25:389-98.

58. Yamada Y, Shigetomi H, Onogi A, et al. Redox-active iron-induced oxidative stress in the pathogenesis of clear cell carcinoma of the ovary. Int J Gynecol Cancer 2011;21:1200-7.

59. Sun D, Li YC, Zhang XY. Lidocaine Promoted Ferroptosis by Targeting miR-382-5p /SLC7A11 Axis in Ovarian and Breast Cancer. Front Pharmacol 2021;12:681223.

60. Zhang Y, Xia M, Zhou Z, et al. p53 Promoted Ferroptosis in Ovarian Cancer Cells Treated with Human Serum Incubated-Superparamagnetic Iron Oxides. Int J Nanomedicine 2021;16:283-96.

61. Greenshields AL, Shepherd TG, Hoskin DW. Contribution of reactive oxygen species to ovarian cancer cell growth arrest and killing by the anti-malarial drug artesunate. Mol Carcinog 2017;56:75-93. 
62. Foulkes WD, Smith IE, Reis-Filho JS. Triple-negative breast cancer. N Engl J Med 2010;363:1938-48.

63. Chen MS, Wang SF, Hsu CY, et al. CHAC1 degradation of glutathione enhances cystine-starvation-induced necroptosis and ferroptosis in human triple negative breast cancer cells via the GCN2-eIF2 $\alpha$-ATF4 pathway. Oncotarget 2017;8:114588-602.

64. Ma S, Dielschneider RF, Henson ES, et al. Ferroptosis and autophagy induced cell death occur independently after siramesine and lapatinib treatment in breast cancer cells. PLoS One 2017;12:e0182921.

65. Zhu HY, Huang ZX, Chen GQ, et al. Typhaneoside prevents acute myeloid leukemia (AML) through suppressing proliferation and inducing ferroptosis associated with autophagy. Biochem Biophys Res Commun 2019;516:1265-71.

66. Hou Y, Cai S, Yu S, et al. Metformin induces ferroptosis by targeting miR-324-3p/GPX4 axis in breast cancer. Acta Biochim Biophys Sin (Shanghai) 2021;53:333-41.

67. Yang J, Zhou Y, Xie S, et al. Metformin induces Ferroptosis by inhibiting UFMylation of SLC7A11 in breast cancer. J Exp Clin Cancer Res 2021;40:206.

68. Calvayrac O, Pradines A, Pons E, et al. Molecular biomarkers for lung adenocarcinoma. Eur Respir J 2017;49:1601734.

69. Yu HC, Chen R, Guo SP, et al. Sulfasalazine induces

Cite this article as: Jiang $M, \mathrm{Hu} R$, Yu R, Tang Y, Li J. A narrative review of mechanisms of ferroptosis in cancer: new challenges and opportunities. Ann Transl Med 2021;9(20):1599. doi: $10.21037 /$ atm-21-4863 ferroptosis of breast cancer ZR-75-1 cells and its mechanism. Tumor 2018;38:27-35.

70. Sukiennicki GM, Marciniak W, Muszyńska M, et al. Iron levels, genes involved in iron metabolism and antioxidative processes and lung cancer incidence. PLoS One 2019;14:e0208610.

71. Wen CP, Lee JH, Tai YP, et al. High serum iron is associated with increased cancer risk. Cancer Res 2014;74:6589-97.

72. Ni J, Chen K, Zhang J, et al. Inhibition of GPX4 or mTOR overcomes resistance to Lapatinib via promoting ferroptosis in NSCLC cells. Biochem Biophys Res Commun 2021;567:154-60.

73. Ma L, Zhang X, Yu K, et al. Targeting SLC3A2 subunit of system XC- is essential for m6A reader YTHDC2 to be an endogenous ferroptosis inducer in lung adenocarcinoma. Free Radic Biol Med 2021;168:25-43.

74. Pattabiraman DR, Weinberg RA. Tackling the cancer stem cells - what challenges do they pose? Nat Rev Drug Discov 2014;13:497-512.

75. Proneth B, Conrad M. Ferroptosis and necroinflammation, a yet poorly explored link. Cell Death Differ 2019;26:14-24.

(English Language Editor: B. Draper) 